\title{
Attribute based Encryption and Decryption Technique
}

\author{
P. Jyothi \\ Asst. Prof.,CSE Dept., \\ PVKK IT,Anantapuramu
}

\author{
D. Raghavaraju \\ Asst. Prof.,CSE Dept., \\ SVIT,Anantapuramu
}

\author{
S. Vasundara, $\mathrm{PhD}$ \\ Professor \& Head of CSE \\ Dept,JNTUA
}

\begin{abstract}
Inside a dispersed foriegn atmosphere, anyone receives a untrusted company which has a change for better crucial allowing this foriegn to turn any kind of $\mathrm{ABE}$ ciphertext fulfilled through the wearer's features. Stability of $\mathrm{ABE}$ technique with outsourced decryption makes certain that a foe (including any destructive cloud) aren't going to be capable to study everything about the encrypted meaning. Anyway, this product doesn't ensure this correctness with the change for better completed through the foriegn. Verifiability ensures if thez change for better offers transpired appropriately or maybe certainly not. With this paper, it all recommend a improvised version of $A B E$ with verifiability. A new concrete scheme pertaining to $\mathrm{ABE}$ with verifiable outsourced decryption can be recommended which is both safe along with verifiable without having based after hit-or-miss oracles.
\end{abstract}

Keywords

$\mathrm{ABE}, \mathrm{CP}-\mathrm{ABE}$, Verifiable C-ABE, Concrete ABE.

\section{INTRODUCTION}

In the allocated setting having untrusted computers for instance cloud, complex-access handle systems are needed gain access to the encrypted files. Attribute Centered Encryption (ABE) is often a fresh community important dependent one-to-many encryption empowering handle around encrypted files making use of access procedures in addition to related features. ABE is often a particular case involving well-designed encryption. In the community important encryption program, files is usually encrypted for being examine by the unique one who has brought any community important. In the well-designed encryption program, the operation $\mathrm{f}(\mathrm{x}$ : $\mathrm{y})$ decides such a person having magic formula important ymca can easily study on any cipher textual content encrypted underneath back button. The boosted operation in addition to mobility offered by this sort of devices can be quite appealing for a lot of useful applications. Presented a lot of the likely uses involving ABE devices, creating useful devices making sure robust protection is surely an crucial matter.

The current $\mathrm{ABE}$ schemes are generally selectively secure which is the protection is usually proved intended for weakened type where an element of the cipher textual content have to be disclosed before the enemy gets the public variables.

In this particular perform, many of us recommend changes for the first $\mathrm{ABE}$ structure to make sure verifiability in addition to recommend any Concrete- $\mathrm{ABE}(\mathrm{C}-\mathrm{ABE})$ structure having verifiable outsourced decryption. The offered structure greatly reduced the working out time period essential for resourcelimited devices to recoup plaintexts.

\section{EXISTINGSYSTEM}

Within the individuality primarily based encryption process, a great expert markets secrets in order to users having affiliated identities, and also mail messages are usually encrypted on to identities. These kind of plans were verified safe from the haphazard oracle product. Selectively safe plans are usually constructed of which confined towards dividing approach in the secrets yet incurs cost regarding significant and also intricate types. Hierarchical Identity Based Encryption (HIBE)[2] stretches your efficiency of individuality primarily based encryption to feature a new hierarchical structure on identities, exactly where identities may delegate secret secrets on their subordinate identities. The guaranteeing request of $\mathrm{ABE}$ is usually flexible gain access to management of encrypted info stored from the cloud, utilizing gain access to rules and also ascribed qualities related to personal secrets and also cipher text messaging. You can find 2 forms of ABE plans:

Key-policy ABE (KP-ABE) and also Cipher text-policy $\mathrm{ABE}(\mathrm{CP}-\mathrm{ABE})$. [1]

Within a CP-ABE plan, every single cipher text is usually related to a great gain access to insurance plan on qualities, and also every single user's personal crucial is usually associated with a list of qualities. The consumer will be able to decrypt a new cipher text provided that your list of qualities for this user's personal crucial fulfills your gain access to insurance plan for this cipher text. Within a KP-ABE plan, your tasks of an attribute established and also a great gain access to insurance plan are usually changed. The prior buildings regarding $\mathrm{ABE}$ plans supply a minimal type of stability the spot that the assailant is needed to declare the marked he or she expects in order to assault ahead of seeing people variables in the process. Your formation in the public variables partitioning your secrets in to 2 courses: people who your simulator can make, and people which might be employed to your simulator in solving the problem. Regarding ABE programs, personal secrets and also cipher text messaging get far more structure so of which different secrets having sharable qualities may be connected which seriously eliminates allowable partitioning.

On the list of effectiveness downsides of the extremely recent $\mathrm{ABE}$ plans is usually of which decryption is usually costly regarding resource-limited units as a result of pairing businesses. Your complexness in the $\mathrm{ABE}$ product grows seeing that the quantity of pairing secrets in order to decrypt your cipher text grows.

To help conquer the issues mixed up in ABE plans, most of us bring in a great increased idea of ABE having outsourced decryption, reducing your decryption overhead for the users.

\section{PROPOSED SYSTEM}

A C-ABE scheme is a ciphertext-policy attribute-based encryption system consists of four algorithms: Setup, Encrypt, KeyGen, and Decrypt. 


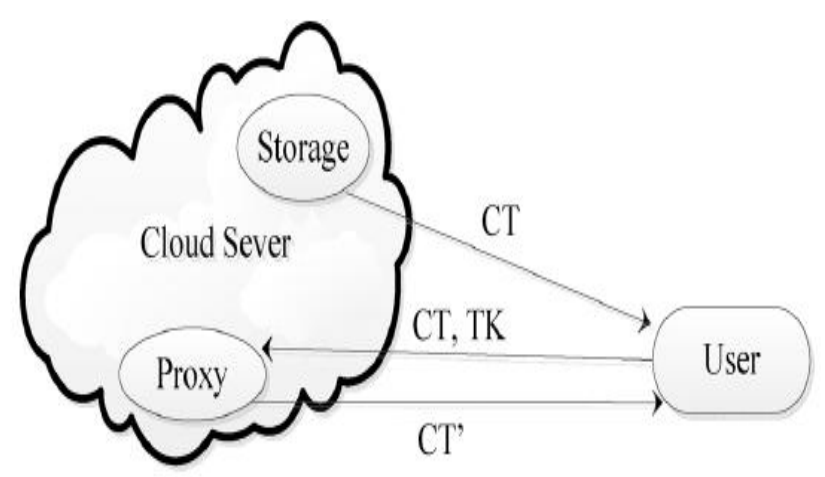

Fig 1: Concrete ABE scheme with outsourced decryption

$\operatorname{Setup}(\lambda, \mathrm{U}) \rightarrow(\mathrm{PK}, \mathrm{MSK}):$ The particular setup algorithm takes in the security parameter $\lambda$ as well as the feature world information $\mathrm{U}$. The idea components the general public guidelines PK and also a get good at technique important MSK.

Encrypt(PK, L, A) $\rightarrow$ CT: The particular encryption algorithm takes in the general public guidelines PK, the concept L, and the admittance composition Some sort of over the world connected with characteristics. The result will be a ciphertext CT so that solely users whose exclusive tips fulfill the admittance composition Some sort of are able to draw out $\mathrm{L}$.

KeyGen(MSK, PK, S) $\rightarrow$ SK: The real key technology algorithm takes in the get good at technique important MSK, the general public guidelines $\mathrm{PK}$, and a collection of characteristics Azines. The idea components an individual important SK.

$\operatorname{Decrypt}(\mathrm{PK}, \mathrm{CT}, \mathrm{SK}) \rightarrow \mathrm{M}$ : The particular decryption algorithm takes in the general public guidelines PK, the cipher textual content CT, and also a exclusive important SK. Should the number of characteristics with the exclusive important satisfies the

admittance composition with the cipher textual content, it components the concept $\mathrm{L}$.

With regard to correctness, it all call for the subsequent to hold:

1) Should the number of characteristics satisfies the admittance composition Some sort of,then L $\leftarrow$ $\operatorname{Decrypt}(\mathrm{Pk}, \mathrm{Sks}, \mathrm{CT})$.

2) Normally, Decrypt $(\mathrm{Pk}, \mathrm{Sks}, \mathrm{CT})$ components the malfunction mark $\lrcorner$.

The particular security explanation with regard to $\mathrm{C}-\mathrm{ABE}$ programs are generally since provided under:

Startup: The particular opposition runs the Startup algorithm and gives the general public guidelines PK for the opponent.

Period 1 The particular opponent concerns the opposition with regard to exclusive tips equivalent to be able to sets connected with characteristics $\mathrm{S} 1, \ldots . ., \mathrm{Sq} 1$.

Difficult task The particular opponent expresses a pair of similar period emails $\mathrm{M} 0$ and $\mathrm{M} 1$ and the admittance composition $\mathrm{A}^{*}$. That admittance composition can not be content through one of the queried feature sets $\mathrm{S} 1, \ldots . ., \mathrm{Sq} 1$. The particular opposition flips the arbitrary coin $\beta \in 0,1$, and encrypts $\mathrm{Mb}$ underneath $\mathrm{A}^{*}$, creating $\mathrm{CT}^{*}$. The idea allows $\mathrm{CT}^{*}$ for the opponent.
Period a couple of The particular opponent concerns the opposition with regard to exclusive tips equivalent to be able to sets connected with characteristics $\mathrm{Sq} 1+1, \ldots . ., \mathrm{Sq}$, with all the added in stops that probably none of the gratify $\mathrm{A}^{*}$.

Think The particular opponent components the speculate $\beta^{\prime}$ with regard to $\beta$.

The benefit of the opponent is usually this particular video game is usually de_ned being $\operatorname{Pr}\left[\beta=\beta^{\prime}\right]-1 / 2$.

a couple of. Most of us remember that the style can easily possibly be extended to address chosen-ciphertext assaults through allowing for decryption concerns throughout Period 1 and Period a couple of.

Definition 3 Some sort of cipher text-policy attribute-based encryption method is usually thoroughly safeguarded in the event that just about all polynomial time period enemies include for the most part the minimal advantage within this security video game.

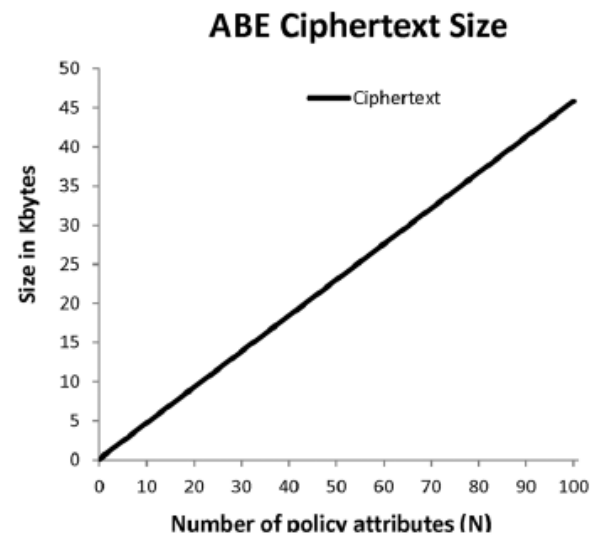

Fig 2: Performance of C-ABE for ciphertext size

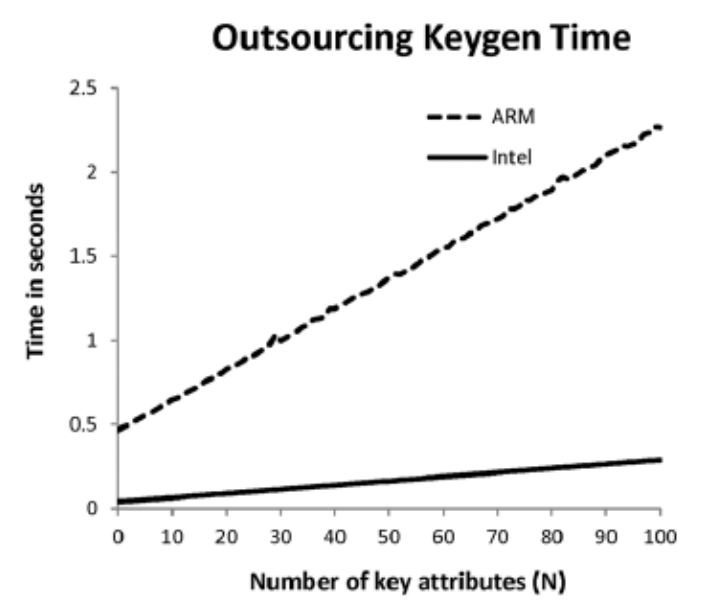

Fig 3: Performance of C-ABE for Outsourced Keygeneration

\section{CONCLUSION}

Your recommended ABE program together with validated outsourcability will not are based upon the particular randomly oracles. Your $\mathrm{ABE}$ ciphertext sizing and decryption/transformation occasion enhance linearly because the cipher text message policy's complexity increases. Your recommended freelancing significantly lowers the particular calculation occasion required for gadgets together with 
limited computing resource to extract the particular plaintext. Your checksum price can be obtained as a determination for the ordinary text message which often can checked out if the change features transpired correctly or definitely not.

\section{REFERENCES}

[1] Junzuo Lai, Robert H. Deng, Chaowen Guan, and JianWeng,"Attribute-Based Encryption With VerifiableOutsourcedDecryption",IEEETRANSACTIO NS ON INFORMATIONFORENSICSANDSECURITY, VOL. 8, NO. 8, AUGUST $2013 \quad 1343$.

[2] A. B. Lewko, T. Okamoto, A. Sahai, K. Takashima, and B. Waters, "Fully secure functional encryption: Attributebased encryption and(hierarchical) inner product encryption," in Proc. EUROCRYPT, 2010, pp. 62-91.
[3] M. Green, S. Hohenberger, and B.Waters, "Outsourcing thedecryption of ABE ciphertexts," in Proc. USENIX Security Symp.,San Francisco, CA, USA, 2011.

[4] C. Dwork, "Differential Privacy: A Survey of Results," Proc. FifthInt'l Conf. Theory and Applications of Models of Computation (TAMC),pp. 1-19, 2008.

[5] M. Green, A. Akinyele, and M. Rushanan, Libfenc: The FunctionalEncryption Library

[6] M. Bellare, A. Boldyreva, and A. Palacio, "An uninstantiablerandomoracle- model scheme for a hybridencryption problem," in Proc. EUROCRYPT, 2004, pp. 171-188. 